\title{
Sistemas de mantenimiento en turismo
}

\author{
Isabel Cristina Parra García*
}

Universidad de las Tunas (Cuba)

\begin{abstract}
Resumen: En Cuba las empresas requieren desarrollar sus procesos productivos y de servicios de forma tal que le permitan tomar las mejores decisiones y las conviertan en organizaciones de excelencia. En un contexto marcado por el crecimiento de la demanda de un elevado nivel de prestación de los servicios se desarrolla la siguiente investigación que tiene como objetivo diagnosticar el sistema de gestión de la calidad en los Servicios Técnicos del hotel Bisas Covarrubias según los requisitos de la norma 9001: 2015, un elemento de vital importancia para alcanzar el nivel más elevado en lo referente a calidad dirigido a los procesos de mantenimiento. En su elaboración se utilizaron técnicas de: recopilación de información y un estudio bibliográfico acerca del tema de investigación. Entre los principales resultados alcanzados con esta investigación se encuentra el nivel de satisfacción del cliente manifestado de satisfactorio en su mayoría.
\end{abstract}

Palabras Clave: Mantenimiento; Turismo; Sistema de gestión de calidad.

\section{Systems of maintenance in tourism}

Abstract: In Cuba, companies need to develop their production and service processes in a way that allows them to make the best decisions and turn them into organizations of excellence. In a context marked by the growth of demand for a high level of service provision, the following research aims at an in-depth analysis of the quality management system in the Technical Services of the Bisas Covarrubias hotel in line with ISO 9001: 2015, an element of vital importance to attain the highest quality in maintenance processes. The article includes a review of the specialist literature on the subject of the investigation. Among the main results achieved with this research is the preceived satisfaction of the client.

Keywords: Maintenance; Tourism; Quality management system.

\section{Introducción}

Este artículo es el resultado de la investigación, Diagnóstico del sistema de gestión de la calidad en el proceso de mantenimiento; caso Hotel Brisas Covarrubias ubicado en Puerto Padre, provincia Las Tunas perteneciente al grupo hotelero Cubanacán S.A se propone realizar un estudio preliminar, que permita mejorar la calidad de los servicios prestados, para la posterior implantación de un Sistema de Gestión de la Calidad basados en las NC ISO 9000 vigentes y normas actuales acordes a las características particulares de la organización. Después de haber realizado un estudio en la entidad se detectaron las siguientes deficiencias (No tienen implementado un SGC basado en las normas ISO, el conocimiento en materia de calidad continúa siendo insuficiente, persisten algunos problemas de infraestructura, insuficientes accesorios y útiles para desarrollar un servicio eficiente, deficiencias en el trabajo con la documentación requerida por la NC ISO 9001:2015, insatisfacciones de los clientes por el servicio recibido.

Por lo anteriormente expuesto, se define como problema de investigación: Insuficiencias del sistema de gestión de la calidad de los procesos operativos en el hotel Brisas Covarrubias.

En correspondencia se asume como objeto de investigación: la gestión de la calidad en los servicios.

Planteando como objetivo general: diagnosticar el sistema de gestión de la calidad en los Servicios Técnicos del hotel Bisas Covarrubias según los requisitos de la norma 9001: 2015

Definiendo como campo de acción: Los Sistemas de Gestión de la Calidad en los Servicios Técnicos.

Master en Ingeniera Industrial; Profesor Asistente - Centro de trabajo: Universidad de Las Tunas; E-mail: ailenet@ult.edu.cu 
Defendiendo la hipótesis: si se diagnostica el SGC en los Servicios Técnicos se podrá contribuir a la mejora de las insuficiencias en el hotel Brisas Covarrubias.

En correspondencia con el objetivo general y con la hipótesis se plantean las tareas de investigación siguientes:

1) Revisión de la bibliografía relacionada con la Calidad en los procesos fundamentales de servicio en el turismo.

2) Determinación de los principales problemas que afectan la Gestión de la Calidad en el hotel Brisas Covarrubias.

- Aplicación parcial del procedimiento seleccionado para el diseño del Sistema de Gestión de la Calidad en el hotel Brisas Covarrubias.

\section{Marco Teórico}

Para el desarrollo de la investigación se tomará como concepto de calidad el referido en la NC ISO 9000:2015 el cual plantea que: una organización orientada a la calidad promueve una cultura que da como resultado comportamientos, actitudes, actividades y procesos para proporcionar valor mediante el cumplimiento de las necesidades y expectativas de los clientes y otras partes interesadas pertinentes.

La gestión es el conjunto de "actividades coordinadas para dirigir y controlar una organización", por lo que puede decirse que la gestión de la calidad es "la gestión de actividades coordinadas para dirigir y controlar una organización con respecto a la calidad". Incluye el establecimiento de las políticas de calidad, los objetivos de la calidad, los procesos para lograr estos objetivos, el aseguramiento de la calidad, el control de la calidad y la mejora de la calidad. (NC ISO 9000/2015)

Para una buena gestión de la calidad se debe determinar y establecer la política y los objetivos de la calidad, los cuales han de estar en concordancia con las características propias de cada organización. Estos objetivos se alcanzan a través de un conjunto de proceso interrelacionados y dependientes: planificación, aseguramiento, control y mejora de la calidad.

El autor considera que la NC ISO 9000:2015 define la gestión de la calidad de forma clara y precisa, por lo cual es adecuado su uso, puesto que, en la gestión de la calidad, dirigir y controlar incluye el establecimiento de la política, los objetivos, así como la planificación, el control, el aseguramiento y la mejora de la calidad, lo que permite una mayor satisfacción de las expectativas de los clientes.

Según la ISO 9000:2015 un SGC comprende actividades mediante las que la organización identifica sus objetivos y determina los procesos y recursos requeridos para lograr los resultados deseados. Gestiona los procesos que interactúan y los recursos que se requieren para proporcionar valor y lograr los resultados para las partes interesadas pertinentes.

Posibilita a la alta dirección optimizar el uso de los recursos considerando las consecuencias de sus decisiones a largo y corto plazo. Proporciona los medios para identificar las acciones para abordar las consecuencias previstas y no previstas en la provisión de productos y servicios.

"En el turismo calidad y servicio son dos categorías que tienen una importancia de primer orden y poseen una relación estrecha, que pudiera dar la impresión de que se trata de lo mismo, pero existen diferencias marcadas entre ellas que no debemos olvidar. La calidad, como la belleza, depende del cristal con que se mira. En el caso del turismo, el producto es con frecuencia intangible y la calidad no resulta aparente o visible hasta después de haberlo consumido. El consumidor no tiene la posibilidad de devolver el producto si la calidad es inferior a la esperada. La producción y el consumo coinciden a menudo en el tiempo ante los ojos de los consumidores, que se encuentran presentes durante el proceso." (Toro Soto, 2011)

En su programa de trabajo, la Organización Mundial de Turismo entiende la calidad del turismo como "el resultado de un proceso que implica la satisfacción de todas las necesidades, exigencias y expectativas legítimas de los consumidores respecto a los productos y servicios, a un precio aceptable, de conformidad las condiciones contractuales mutuamente aceptadas y con los factores subyacentes que determinan la calidad tales como la seguridad, la higiene, la accesibilidad, la transparencia, la autenticidad y la armonía de una actividad turística preocupada por su entorno humano y natural”.

Para lograr la calidad de servicio en el ámbito turístico, es necesario implementar una orientación hacia los clientes, quienes a fin de cuentas, son los que evalúan y deciden si pagarán por un servicio.

\section{Metodología}

Se analizó la metodología de Marilet Cazañas Rivero de La Habana: Instituto Superior Politécnico José Antonio Echeverría (CUJAE) (2011), Diseño de un sistema de gestión de la calidad en el proceso 
de alojamiento en el hotel "gran Caribe Villa Tortuga: esta fue la principal bibliografía tomada como referencia para realizar el procedimiento ya que está enfocada en un proceso operativo y es una de las propuestas más completas, consta con 10 actividades teniendo en cuenta el ciclo Deming, la misma se puede aplicar a cualquier organización. Es importante destacar que este modelo parte de la realización de un diagnóstico de la situación actual de la instalación, que evidencie la necesidad de aplicar un SGC.

Otra propuesta analizada fue la Metodología para Implementar la Gestión de la Calidad y la Reingeniería de procesos de la MSc. Iliana Hernández Concepción de la Universidad de Holguín basada en las normas ISO 9000 del 2000. Con su aplicación se logra inicialmente, determinar los procesos (estratégicos, operativos y de apoyo) que se desarrollan en la organización para garantizar la calidad, caracterizando cada uno de ellos con la ficha de procesos. Este análisis detallado permite realizar un diagnóstico profundo, identificando las dificultades que presenta cada proceso, así como los cambios que se deben emprender, que incluye el diseño o rediseño de los procesos actuales del sistema. Además, se concibe el proceso de cambio organizacional necesario, el plan de formación para todo el personal y se elabora la documentación que sustenta el funcionamiento del SGC, lo cual parte de un análisis profundo del cumplimiento de los principios de la gestión de la calidad arraigada en la organización. Finalizado el proceso de diseño del sistema, se comienza con la implantación y la realización de auditorías hasta la certificación.

También fue examinado el Procedimiento para el diseño e implantación del sistema integrado de gestión de la calidad, la seguridad y salud en el trabajo y la protección medioambiental propuesto por el Ing. Martín Alberto Ayala López de la Universidad de Holguín, el cual cuenta con siete fases que van desde el involucramiento hasta las necesidades de acciones de mejora.

Luego de revisar las mismas se llegó a las siguientes conclusiones:

- El enfoque de gestión más utilizado es el normalizado.

- La mayoría incluye etapas que van desde el diagnóstico al seguimiento, control y mejora.

- Con la actualización de la NC ISO 9001:2015 las metodologías y procedimientos existentes se encuentran obsoletos por no mostrar un enfoque basado en riesgos que permita trazar acciones preventivas para lograr la conformidad de productos y servicios.

Es por ello que el autor asume la propuesta realizada por Lisbet Toranzo Rodríguez en su tesis de grado con el tema "Procedimiento para el diseño del Sistema de Gestión de la Calidad en el proceso operativo de restauración en el Hotel Brisas Covarrubias" donde se presenta un procedimiento para la implementación de un SGC enfocado a procesos. Como todo procedimiento comienza con un diagnóstico de la organización para conocer el estado actual de la misma y hacia donde deben de estar dirigidos nuestros esfuerzos. Las etapas de este procedimiento tienen relación con el ciclo de Deming.

\section{Explicación de las etapas del procedimiento}

\subsection{Diagnóstico de la gestión de la calidad}

Paso 1: Análisis de la necesidad de la organización de implantar un SGC: el investigador debe realizar este paso mediante entrevistas con el encargado de atender la calidad y el director de la organización para corroborar que existe realmente una necesidad.

- Creación del grupo de experto: en este punto se determina quien conformará el grupo de experto escogiendo los que dirigen cada proceso, el encargado de la calidad y otras personas que el investigador considere conveniente que desempeñen un papel clave dentro de la organización. Este grupo debe estar conformada al menos por 5 personas.

Paso 2: Caracterización de la organización: en este paso se debe conocer brevemente su localización geográfica, su misión, visión, objeto social, política y objetivos de calidad, además de cualquier otro aspecto que el investigador considere relevante. Se debe describir todos estos elementos para conocen las características de la organización.

- Caracterización del proceso operativo: en este punto se describe brevemente el proceso operativo que será objeto de investigación. Se debe describir como está estructurado organizativamente (organigrama) el proceso, como se divide, la formación profesional del personal, los principales clientes y proveedores.

- Explicación del proceso: en este aspecto se debe describir la secuencia de actividades que se realizan en el proceso mediante un diagrama OTIDA. 
- Análisis económico de los costos del proceso: se realizará un breve análisis tomando como muestra un período de tiempo (mes, trimestre, año) analizando los gastos en que se incurrieron para realizar el proceso.

- Paso 3: Análisis interno y externo: en este paso se realizará un levantamiento de los principales resultados que se han obtenido hasta este punto del diagnóstico y otros elementos que el grupo de expertos considere necesario para posteriormente elaborar la matriz DAFO.

- Análisis de los factores: se escogerá una guía para realizar el análisis de los factores externos.

a) Determinación y análisis de las no conformidades en el proceso: se hace un levantamiento de las principales insuficiencias detectadas en el proceso para buscar las causas que las originan mediante un diagrama Ishikawa.

b) Encuesta a los clientes mediante el método SERVQUAL: se elaborarán las encuestas utilizando este método con sus 5 dimensiones.

c) Encuesta a los trabajadores para verificar el cumplimiento de los principios de gestión de la calidad: se elaborarán las encuentras formuladas con los principios de gestión de la calidad de la familia de normas 9000 vigentes para corroborar que la organización los cumple adecuadamente.

d) Encuesta a los proveedores: se realizarán encuestas a los proveedores para medir si la relación de ellos con la organización es buena, regular o mal.

Paso 4: Aplicación de la lista de chequeo basado en la NC ISO 9001:2015.

Paso 5: Construcción de la MEFI, MEFE y la matriz DAFO.

Paso 6: Propuesta de soluciones.

Definición de las funciones del equipo de trabajo (fue creado en el diagnóstico) que van propiciar el desarrollo del procedimiento.

\subsection{Identificación y secuenciación de los procesos (subprocesos):}

En esta etapa se pretende mediante entrevistas con el equipo de trabajo identificar los procesos que intervienen en la organización, así como hacer un desglose de los subprocesos que actúan dentro del proceso operativo que se esté diseñando.

a) Elaboración del mapa de proceso.

b) Elaboración del macroproceso.

\subsection{Descripción del proceso que se diseña}

a) Elaboración de los diagramas de flujos (en el caso que se pueda).

b) Confección de la ficha de proceso (son las características de los procesos).

c) Identificación, evaluación y tratamiento de los riesgos de calidad por la NC ISO 31000:2009.

\subsection{Identificación de las necesidades del cliente externo}

a) Investigación de las nuevas tendencias del proceso (en esta subetapa se pretende hacer un estudio para conocer las tendencias que tiene hoy día el proceso e identificar las preferencias de los clientes).

b)Aplicación de encuestas para medir el Índice de Percepción de la Calidad del Servicio (IPCS) y el nivel de satisfacción del cliente (NSC).

c) Análisis de las principales quejas de los clientes. (Las que se obtuvieron como resultado de las encuestas para este análisis se utilizará el diagrama Paretto).

\subsection{Capacitación y formación de personal de la organización}

Esta necesidad surge de una de las fases del diagnóstico.

a) Planificar conferencias, cursos, seminario referente al SGC.

b) Ejecutar plan de capacitación.

c) Evaluar el impacto de la capacitación: este aspecto se puede medir mediante encuestas y exámenes.

\subsection{Documentación del SGC}

a) Descripción y análisis de los puntos que compone el manual de calidad (verificar que la política y objetivos de calidad se correspondan con lo establecido en la norma 9001: 2015).

b) Analizar y organizar los procedimientos, instrucciones y registros que debe contener la estructura documental del proceso. 


\subsection{Gestión de los procesos.}

a) Medición y seguimiento de los procesos: establecer los indicadores que permita medir el proceso, sugiriéndole a la organización herramientas que el investigador considere que sea útil.

b) Auditar los procesos: este aspecto lo lleva a cabo los auditores internos o los que vayan a certificar el SGC.

\subsection{Mejora}

a) Analizar las causas que provocan las no conformidades que se obtuvieron como resultado de las auditorías a los procesos.

b) Plantear acciones correctivas y preventivas para minimizar las causas de las no conformidades.

c) Verificar el cumplimiento de las acciones.

d) Elaborar un plan de mejora.

\section{Resultados}

Etapa 1: Análisis de la necesidad de la organización de implantar un SGC.

El objetivo principal de esta investigación es brindar elementos para la crear las bases necesarias para la posterior implementación del SGC en el Hotel Brisas Covarrubias. Después de dialogar con la directora y la técnica de calidad se constató que el hotel tiene la necesidad de realizar dicha acción ya que gracias a la aplicación de un sistema de gestión de calidad, se consigue reducir los errores durante el proceso de prestación de servicios, lo cual genera una disminución de los costos y a su vez, el incremento de la productividad. En consecuencia positiva, se obtiene la satisfacción de los clientes, así como, la elevación moral de los trabajadores. El resultado final es el fortalecimiento de la competitividad del hotel mediante el aumento de las utilidades en un corto plazo y un mayor posicionamiento en el mercado. Para la implementación del SGC del Hotel es necesario la realización del diseño del SGC en cada uno de los procesos, en esta primera etapa del procedimiento se pretende realizar un diagnóstico en la gestión de la calidad en el proceso de mantenimiento y luego diseñar dicho sistema, por lo que es imprescindible hacer análisis de las principales deficiencias en el proceso antes mencionado que impiden al hotel implementar el SGC según la NC 9001:2015.

Paso 1: Creación del grupo de experto.

1. Definir el personal que participará directamente en el estudio.

Con el conocimiento de la necesidad del estudio y la motivación del personal para su realización, se procede a la creación del Grupo de Experto, que está conformado por el personal de la organización que organizará y ejecutará todas las tareas. En función de la complejidad de la organización este grupo podrá tener más o menos integrantes debiendo estar representadas todas las áreas afectadas.

Para la creación del grupo de expertos se propone utilizar el procedimiento de Hurtado. Los especialistas se seleccionan por los conocimientos específicos y la calificación técnica, debido a la influencia que tienen en la consistencia de los resultados que se desean.

A los efectos de este trabajo se han considerado como requisitos para que una persona sea seleccionada especialista:

- Prestigio y profesionalidad reconocidos en la sociedad.

- Relación laboral, docente o práctica, con la gestión de la calidad y su visión con una perspectiva turística.

- La representatividad de los lugares de procedencia. (Se considera así que dentro del grupo de especialistas deben estar representados: teóricos y prácticos).

Para la obtención de la información necesaria para la selección de los especialistas, se utilizó una encuesta. Además, se calculan el Coeficiente de Competencia (K) de cada especialista en función del Coeficiente de Conocimiento o Información (Kc) y el Coeficiente de Argumentación (Ka) según el procedimiento de Hurtado (2003).

Para validar esto se aplica la fórmula siguiente:

$\mathrm{K}=1 / 2(\mathrm{Kc}+\mathrm{Ka})$

La literatura consultada plantea que el coeficiente de competencia del especialista debe comportarse en el rango $0.8 \leq \mathrm{k} \leq 1.0$. 
2. Describir las funciones del equipo de trabajo.

Cada integrante del grupo juega un papel primordial en el proceso. Para una mejor organización del mismo se recomienda definir previamente cuáles son las tareas que le corresponde desarrollar a cada uno de los miembros.

3. Capacitar al personal que conforma el grupo.

Lograr la formación de un personal competente, con el conocimiento necesario para interactuar con los procedimientos de los SGC, pudiendo interpretar sus términos, definiciones y aplicar políticas establecidas, según corresponda a cada caso es una tarea de gran relevancia. El personal escogido para integrar el grupo de expertos debe tener un conocimiento amplio que le permita aplicar correctamente las técnicas y herramientas para así desarrollar con éxito cada una de las etapas a de la implementación. Las principales temáticas a tratar durante la capacitación están relacionadas con la familia de normas cubanas ISO 9000 haciendo énfasis en el proceso de implantación del SGC. También se debe preparar al personal en lo referente a las distintas técnicas y herramientas a utilizar durante el proceso.

Planificar conferencias, cursos, seminario referente al SGC: La capacitación ha de llevarse a cabo de una forma bien organizada y en unión con los organismos competentes que ayuden a la organización a realizar la misma de la forma más adecuada brindando los conocimientos necesarios con la calidad y profundidad requerida. Se ha de planificar un plan de capacitación que incluya cada una de las actividades necesarias. En el mismo se incluirá la fecha de realización, las personas involucradas, el tipo de actividad a desarrollar.

Ejecutar plan de capacitación: No es más que llevar a cabo las actividades proyectadas en el plan.

4. Evaluar el impacto de la capacitación.

Una vez realizada la capacitación se hace imprescindible evaluar el alcance que tuvo para conocer si fueron satisfechas las expectativas y si el grupo de expertos está listo para continuar avanzando en el arduo proceso. Se ha de definir si las acciones ejecutadas están en correspondencias con las regulaciones del objetivo del proceso.

Evaluar el nivel de conocimiento alcanzado por los integrantes durante el desempeño de sus funciones, inculcándoles que la respuesta va a estar dada en la sistematicidad y en la elevación de su formación cultural.

Se creó un grupo de experto, para dar cumplimento a esta subetapa dicho grupo tendrá la responsabilidad de estar presentes y disponibles durante el desarrollo del procedimiento, quedando conformado de la siguiente manera:

- Director General:

- Subdirector Comercial y Relaciones:

- Técnico de Control de la Calidad:

- Subdirector de Recursos Humanos:

- Jefe de Servicios Técnicos:

- Especialista de Servicios Técnicos:

\section{Etapa 2: Caracterización de la organización}

Brisas Covarrubias se encuentra situada en la costa norte de la provincia de Las Tunas, en el municipio de Puerto Padre. Inicialmente, desde su fundación el 18 de diciembre de 1998, perteneció al Grupo Hotelero Gran Caribe, con el nombre Comercial Villa Covarrubias hasta el 6 de diciembre del 2005 que pasó al Grupo Hotelero Cubanacán S.A, operando en la modalidad todo incluido categorizado como un Hotel marca Brisas 4 estrellas, adquiriendo el nombre de Brisas Covarrubias para una mejor comercialización del mismo. El Hotel está destinado a la prestación de servicios de hospedaje, alimentación y recreación al Turismo Internacional proveniente de Canadá fundamentalmente ya que el mismo constituye uno de los principales mercados.

La misión de Brisas Covarrubias recoge aspectos significativos como las características propias de nuestro producto, ámbito de actuaciones e influencia, valores y aspiraciones de la organización. Playa Brisas Covarrubias es un polo turístico abierto al turismo nacional e internacional concebido para aprovechar y explotar los intereses turísticos de la provincia y el país en la región localizada en el litoral norte del Balcón del Oriente cubano y caracterizada como un paraíso de aguas transparente y fondos marinos virginales.

Brisas Covarrubias ofrece un disfrute de tranquilidad inigualable para la opción del turismo de familia, rodeado de una vasta naturaleza costera y un ambiente familiar esplendido, que acoge a todo cliente que llega a la instalación recibiendo una amplia propuesta de actividades de animación y deportes náuticos y otros servicios de alta calidad, apoyado en un colectivo de trabajadores y jóvenes y profesionales, con una tecnología de avanzada y un crecimiento económico sostenido, aunque marcamos 
una diferencia con otros hoteles de máxima calidad avanzamos para que la instalación sea competidor digno en la complacencia del cliente en Cuba.

La visión de Brisas Covarrubias es estar entre los preferidos de playa del Grupo hotelero Cubanacán en la isla de Cuba, ofrecer un producto diferente y variado, y con modalidades que compitan con los polos turísticos de Santa Lucía y Guardalavaca y otros del área del Caribe, así como ampliar la capacidad hotelera del lugar.

Política de Calidad:

El hotel Brisas Covarrubias establece como política de calidad, velar en función de la satisfacción y expectativas de los clientes, sustentados en la hospitalidad, iniciativas, los principios de calidad a seguir y el sentido de la responsabilidad, asegurar que todos los trabajadores estén provistos de la formación y los recursos necesarios, a través de la implantación progresiva de un Sistema de Gestión de la Calidad, sostenido en la mejora continua de sus estándares, según se establece en la norma ISO 9001: 2015.

Paso 2: Caracterización del proceso de apoyo de mantenimiento

El área de mantenimiento cuenta con 11 trabajadores de los cuales 2 son directivos. De ellos 3 son graduados del nivel superior, 4 son técnico medio y 4 obreros calificados. Para una correcta ejecución de dicho proceso se cuenta con una estructura organizativa que está compuesta por: Jefe de Mantenimiento de la Instalación, Especialista de Mantenimiento y Operarios de Mantenimiento.

La Misión Fundamental del Departamento de Mantenimiento se centra en; Organizar, Controlar y Desarrollar cualitativa y cuantitativamente los medios y servicios técnicos, para asegurar el funcionamiento ininterrumpido de las operaciones de su Instalación garantizando el confort y la satisfacción plena del Cliente a través de un servicio de esmerada calidad.

Los Objetivos del Departamento de Mantenimiento, estarán encaminados al Control Técnico de la actividad de mantenimiento, tanto de la Instalación como de los medios, es decir, máquinas, equipos y otros.

Dentro de los Objetivos están igualmente contenidos los Principios para el perfeccionamiento de la actividad, el lograr altos niveles de eficiencia tanto en la explotación como en el rendimiento, así como alcanzar resultados que se reflejen en la evaluación de la calidad del servicio.

El Departamento participará en el control, la evaluación y aprobación de las obras que se planteen en la Instalación y que contribuyan a la elevación del nivel de servicios de éstas, a la vez que mejoren la calidad técnica de las mismas.

Por otra parte le corresponde a este Departamento el estar actualizado en técnicas de equipamientos y mantenimiento al igual que servicios especializados que ayuden a su instalación en la elevación del nivel de servicios y confort.

Etapa 3: Análisis interno y externo

Análisis de los factores: este punto no se desarrolló en esta investigación pues en el hotel ya fue aplicada en otra investigación precedente a la actual la guía para el análisis externo donde se realizó un diagnóstico general a la organización la autora es. (Almaguer de Miguel Anaisi. 2015)

Para la realización de este punto fue necesario la aplicación del diagrama Ishikawa para una mejor comprensión y análisis de las causas verdaderas de las no conformidades que existen en el proceso.

Etapa 4: Aplicación de la lista de chequeo

- Contexto de la organización

La organización determina las cuestiones externas e internas que son pertinentes para su propósito y su dirección estratégica y realiza el seguimiento y la revisión de la información sobre las mismas. Determina las partes interesadas que son pertinentes al sistema de gestión de la calidad así como los requisitos, realizando el seguimiento y la revisión de la información.

La entidad objeto de estudio no tiene diseñado, implementado y certificado un SGC. Para gestionar la calidad se utiliza la inspección de rutina por el técnico de control de calidad mediante el cual es posible detectar problemas. Se tienen identificados los procesos del hotel los cuales se pueden apreciar en el mapa de procesos. Cuentan con los criterios y métodos de operación y control de estos procesos los cuales aparecen en el manual de procedimientos. No están disponibles todos los recursos necesarios para apoyar la operación y el seguimiento de los procesos, los resultados de los análisis se evalúan semanalmente. Para alcanzar los resultados planificados de estos procesos se llevan a cabo chequeos diarios y planes de acción donde se vela por el cumplimiento de estas medidas asignando responsables. Se lleva a cabo un proceso de retroalimentación de la satisfacción del cliente para lograr la mejora continua. La organización no aborda los riesgos y oportunidades que deben ser determinados de acuerdo con los requisitos. Mantiene y conserva la información documentada para apoyar la operación de sus procesos para tener la confianza de que los mismos se realizan según lo planificado a excepción del subproceso de ama de llaves.

- Liderazgo 
La alta dirección manifiesta el liderazgo, compromiso e implicación necesaria para el desarrollo e implantación del Sistema de Gestión de Calidad y la mejora continua. No promueve el pensamiento basado en riesgos. No comunica la importancia de una gestión de la calidad eficaz y conforme con los requisitos del sistema de gestión de la calidad. La alta dirección demuestra liderazgo y compromiso con respecto al enfoque al cliente. Determina, comprende y cumple regularmente los requisitos del cliente y los legales y reglamentarios aplicables, manteniendo el enfoque en el aumento de la satisfacción del cliente. No determinan ni consideran los riesgos y oportunidades que pueden afectar a la conformidad de los productos y servicios y a la capacidad de aumentar la satisfacción del cliente.

La alta dirección establece, implementa y mantiene una política de calidad apropiada al propósito y contexto de la organización, que proporciona un marco de referencia para el establecimiento de los objetivos de la calidad e incluye un compromiso de cumplir los requisitos aplicables y de mejora continua. La política de la calidad está disponible y se mantiene como información documentada, se entiende y se aplica dentro de la organización pero no se comunica.

La alta dirección se asegura de que las responsabilidades y autoridades para los roles pertinentes se asignen, se comuniquen y se entiendan en toda la organización asignando la responsabilidad y autoridad para que el sistema de gestión de la calidad sea conforme con los requisitos de la Norma Internacional; informar, en particular, a la alta dirección sobre el desempeño del sistema de gestión de la calidad y sobre las oportunidades de mejora y asegurarse de que se promueve el enfoque al cliente en toda la organización.

- Planificación

La organización establece los objetivos de la calidad para las funciones y niveles pertinentes y los procesos necesarios manteniendo la información documentada sobre los mismos. Estos son coherentes con la política de calidad, son medibles, tienen en cuenta los requisitos aplicables, son pertinentes para la conformidad de los productos y servicios y para el aumento de la satisfacción del cliente, son objeto de seguimiento, se actualizan según corresponda pero no se comunican.

Al planificar cómo lograr sus objetivos de la calidad, la organización determina qué se va a hacer; qué recursos se requerirán; quién será responsable; cuándo se finalizará y cómo se evaluarán los resultados.

- Apoyo

La organización considera las capacidades y limitaciones de los recursos internos existentes y qué se necesita obtener de los proveedores externos.

La organización determina las personas necesarias para la implementación eficaz de su sistema de gestión de la calidad y para la operación y control de sus procesos pero no los proporciona, ya que faltan auditores internos.

La organización no determina, proporciona y ni mantiene la infraestructura necesaria para la operación de sus procesos ya que dependen de la inmobiliaria, pero si garantiza el ambiente necesario para la operación de sus procesos y para lograr la conformidad de los productos y servicios.

Faltan los recursos necesarios para asegurarse de la validez y fiabilidad de los resultados cuando se realiza la medición de los productos y no se aseguran de que los recursos proporcionados sean apropiados para el tipo específico de actividades de seguimiento y medición realizadas. La organización conserva la información documentada apropiada como evidencia de que los recursos de seguimiento y medición son idóneos para su propósito.

El equipo de medición se calibra y verifica anualmente. Se identifican para determinar su estado y se protegen contra ajustes, daño o deterioro que pudieran invalidar el estado de calibración y los posteriores resultados de la medición. La organización determina si la validez de los resultados de medición previos se ha visto afectada de manera adversa cuando el equipo de medición se considere no apto para su propósito previsto, y toma las acciones adecuadas cuando es necesario.

La organización determina los conocimientos necesarios para la operación de sus procesos y para lograr la conformidad de los productos y servicios. Estos conocimientos se mantienen y ponen a disposición. No en todos los casos se determina cómo adquirir o acceder a los conocimientos adicionales necesarios y a las actualizaciones requeridas ya que se evidencia falta de capacitación en los trabajadores.

La organización no se asegura de que los trabajadores sean conscientes de la política ni de los objetivos de la calidad pertinentes.

\section{- Operación}

La organización cumple con los requisitos de prevención de los productos y servicios que se le van a ofrecer a los clientes y determina los recursos necesarios para lograrlo. Falta información documentada 
de que los procesos han sido llevado a cabo y que cumplen con los requisitos de comunicación con los clientes, aunque se les informa todo lo relativo a los productos y servicios, se responden a los pedidos, las consultas, las quejas y en caso de que sea necesario incluye los cambios, además la organización realiza revisiones antes de comprometerse a suministrar productos y servicios y se asegura de cumplir con las especificaciones de los clientes, los no establecidos por ellos pero necesarios para su uso.

En caso de que exista problema entre los requisitos de contrato y pedido la organización se encarga de resolverlo de la mejor manera posible. No se actualiza la información documentada en caso de que haya modificaciones en los requisitos de los productos y servicios, tampoco se comunica a las demás personas interesadas. Cuando se suministra un producto o servicio de proveedores externos la organización se asegura de que se cumplan con los requisitos, que se incorpore dentro de los propios productos y servicios de la organización. Nunca los productos y servicios son suministrados directamente al cliente por parte de los proveedores externos y no están involucrados con los procesos ni toman parte en ellos. La organización no determina, ni aplica criterios para la selección y evaluación de proveedores.

- Evaluación del desempeño

La organización realiza el seguimiento de las percepciones de los clientes del grado en que se cumplen sus necesidades y expectativas y determina los métodos (encuestas) para obtener, realizar el seguimiento y revisar esta información. Analiza y evalúa los datos y la información apropiados que surgen por el seguimiento y la medición.

La organización planifica y establece programas de auditoría, selecciona los auditores, define los criterios de la auditoría y el alcance; pero no los implementa.

Conserva información documentada como evidencia de la implementación de programas y de los resultados de auditorías desactualizadas.

La organización determina y selecciona las oportunidades de me jora e implementa cualquier acción necesaria para cumplir los requisitos del cliente y aumentar la satisfacción mejorando los productos y servicios para cumplir los requisitos, teniendo en cuenta las necesidades y expectativas futuras para corregir, prevenir o reducir los efectos no deseados.

Cuando ocurre una no conformidad, incluida cualquiera originada por quejas, la organización reacciona y, cuando sea aplicable toma acciones para controlarla y corregirla haciendo frente a las consecuencias. Evalúa la necesidad de acciones para eliminar las causas de la no conformidad, con el fin de que no vuelva a ocurrir, mediante la revisión y el análisis de la no conformidad; la determinación de las causas; y la determinación de si existen no conformidades similares, o que potencialmente puedan ocurrir; implementa cualquier acción necesaria y revisa la eficacia de cualquier acción correctiva tomada. Las acciones correctivas son apropiadas a los efectos de las no conformidades encontradas.

La organización conserva información documentada como evidencia de la naturaleza de las no conformidades y cualquier acción tomada posteriormente y los resultados de cualquier acción correctiva, pero esta información se encuentra desactualizada. La organización considera los resultados del análisis y la evaluación, y las salidas de la revisión por la dirección, para determinar si hay necesidades u oportunidades.

Etapa 5: Construcción de la MEFI, MEFE Y la matriz DAFO

Se realizó un intercambio con el grupo de expertos para determinar los factores internos y externos que interactúan dentro del proceso para luego darle la evaluación correspondiente a cada uno.

Según el resultado obtenido en los factores internos indica que en el proceso de mantenimiento predominan las fortalezas por encima de las debilidades pero sin embargo en cuanto a los factores externos se encuentran en una posición desventajosa ya que predominan las amenazas más que las oportunidades. Por tanto se confecciona la matriz DAFO para obtener el cuadrante en que se encuentra el proceso lo que proporcionará la estrategia que se debe seguir.

Etapa 6: Propuesta de soluciones.

A partir del diagnóstico al proceso operativo de mantenimiento en el Hotel Brisas se propone:

- Solicitud de cursos de capacitación a la ONN

- La capacitación de los trabajadores del área y los directivos del hotel, en temas de mantenimiento y SGC.

- Realizar estudios de dirección estratégica en el área de mantenimiento y en el hotel.

- Revisar el proceso de selección de recursos humanos 
2. Definición de las funciones del equipo de trabajo: fue necesario realizar un desglose de los trabajadores que conforman el equipo de trabajo con las funciones que deben realizar para lograr el diseño del SGC en el proceso operativo de restauración.

Identificación y secuenciación de los procesos: el hotel cuenta con tres procesos operativos fundamentales alojamiento, restauración y animación, los cuales conforman la esencia del servicio, a su vez, existen otros procesos que son muy importantes para el funcionamiento de estos tres, dentro de ellos se encuentran los estratégicos y los de apoyo. Dentro de los procesos de apoyo se encuentra el de mantenimiento, el cual es de gran relevancia ya que está relacionado directamente con el cliente. El mismo se compone por:

Jefe de Mantenimiento de la Instalación, Especialista de Mantenimiento, Operarios de Mantenimiento y los talleres.

4. Descripción del proceso que se diseña

a) En la etapa del diagnóstico se describió el proceso según lo tenía el hotel, para una mejor comprensión fue necesario proponer una descripción más detalla del área que compone el proceso por lo que se elaboró un diagrama OTIDA de forma más desglosada.

b) Ficha de Trabajo

Para el mejor desarrollo del MPP, resulta imprescindible que los trabajos a realizar por mantenimiento se controlen siempre mediante "Orden de Trabajo", consistiendo su utilidad en que permite determinar y señalar oportunamente, antes de realizar cada trabajo, todos sus aspectos y controlar, una vez ejecutados, su cumplimiento en detalles a los efectos económicos, estadísticos, técnicos, etc... y permitirá tener un registro-control los materiales utilizados por imprevistos o planificados.

c) Los principales riesgos del área son:

Existe suciedad y pestilencia debido a que el sistema de evacuación de residuales es insuficiente, en el Ranchón ubicado en la playa.

Riesgo de contacto eléctrico en las áreas exteriores debido a la existencia de cables sin soterrar y desprotegidos cerca de los Bungalow.

Riesgo de contacto eléctrico en el área de la discoteca ya que los registros eléctricos no tienen la protección necesaria.

Acciones para darle tratamiento a los riesgos identificados:

Para darle solución a los problemas de pestilencia y suciedad del ranchón de la playa se propone que se realicen las conexiones de los sistemas residuales a los registros.

Para solucionar el riesgo de contacto eléctrico en áreas aledañas a los Bungalow se propone que se terminen de soterrar y proteger los cables.

Se propone para eliminar el riesgo de contacto eléctrico en el área de la discoteca darle la debida protección a los registros electicos.

5. Identificación de las necesidades del cliente externo

a) Investigación de las nuevas tendencias del proceso: Para el desarrollo de este punto fue necesario investigar para conocer las nuevas tendencias que están marcando el mantenimiento, para en el plan de mejora poder brindarle al hotel ideas nuevas y renovadoras que estén encaminadas a lograr la satisfacción del cliente.

Tendencias:

- Confianza en la Relación Cliente - Proveedor

- Agregar Valor al Negocio a Partir de la Especificación del Servicio (Especialización)

- Impulso de I+D+i (Tendencia Innovadora)

- Tendencia Sistemática

- Tendencia Tradicional

- Adecuada Gestión Hacia la Utilización Óptima de los Recursos

- Atributos especialmente valorados ahora y probablemente en el futuro

- Rapidez

- Flexibilidad de horarios

- Lugar agradable

- Variedad

- Amabilidad

- Garantía de Calidad

- Innovación

- Información 
b) Para medir el Índice de Percepción de la Calidad del Servicio (IPCS) y el nivel de satisfacción del cliente (NSC) fue necesario aplicar encuestas, por lo que primeramente se calculó el número de clientes a encuestar arrojando una muestra de 22 , se determinó mediante la expresión matemática reportada por Athanassopoulus y Gounaris (2001).

Los resultados fueron positivos en su mayoría, solo resaltaron cuatro aspectos que influyeron de forma negativa. Se determinó que el servicio presado tiene calidad pero existen insuficiencias las cuales no permiten alcanzar la excelencia la cual representa el nivel más elevado de calidad.

c) Luego de la aplicación de encuestas a los clientes se detectaron como los indicadores fundamentales q afectan la calidad del proceso la pintura, carpintería, telefonía y televisión las cuales influyeron de forma más negativa, entre otras, que representaron más del $36 \%$ de clientes los cuales le dieron una calificación baja.

6. Capacitación y formación de personal de la organización

La capacitación es una de las funciones clave de la administración y desarrollo del personal en las organizaciones, es muy importante para elevar la eficiencia por lo que no debe ser vista como un costo sino como una inversión. Durante la investigación se identificó que los trabajadores carecen de conocimiento sobre el SGC por lo que se realizó un levantamiento de las principales necesidades y se confeccionó un plan para formar a algunos trabajadores para que estos una vez capacitados le proporcionen sus conocimientos al resto.

7. Documentación del SGC

a) Se revisó la política de calidad plasmada en el manual donde se constató que es apropiada al contexto de la organización y apoya su dirección estratégica; además proporciona un marco de referencia para el establecimiento de los objetivos de la calidad e incluye un compromiso de cumplir los requisitos aplicables y de mejora continua. Además analizaron los objetivos de calidad donde se puede afirmar que son coherentes con la política, son medibles, pertinentes para la conformidad de los productos y servicios y para el aumento de la satisfacción del cliente y se actualizan según corresponda.

b) Se realizó una exhaustiva revisión de la documentación del proceso, identificándose varias insuficiencias relacionadas con la no coincidencia de la numeración de los documentos con la descripción del proceso. Todos los documentos fueron acomodados y actualizados por las referencias plasmadas en la ficha de proceso de mantenimiento.

8. Gestión de los procesos

a) La medición y seguimiento de los procesos son esenciales para saber el estado actual en el que se encuentra la organización respecto al que se desea estar, por tanto algunos de los métodos que se le proponen a la técnica de calidad y al encargado directo del proceso de mantenimiento son:

- Hacer más uso de la herramienta del benchmarking.

- Utilizar una vez al mes por el periodo de implantación y certificación del SGC las encuestas confeccionadas en la investigación ya que son una versión mejorada de las que aplica el hotel, ambas están basadas en la herramienta SERVQUAL.

- Auditar más seguido el proceso.

A pesar de no poder culminar el desarrollo del procedimiento por necesitar el involucramiento de auditores externos a la organización, se puede hasta este punto minimizar las no conformidades obtenidas a través de un plan de mejora, donde las acciones propuestas están encaminadas hacia la mejora continua.

\section{Valoración del impacto económico de la investigación}

La instalación considerará las diferentes operaciones y actividades que influyan en sus impactos ambientales significativos cuando desarrolle o modifique controles y procedimientos operacionales. Tales operaciones y actividades pueden incluir: investigación y desarrollo, compras, contratos, manipulación y almacenamiento de productos, actividades de mantenimiento, transportación, comercialización, publicidad, servicio al cliente, compras, entre otras que se estimen pertinentes.

La investigación desarrollada es de gran relevancia para el Hotel Brisas Covarrubias ya que hoy cuentan con un mapa de proceso, diagramas OTIDA, organigramas, los riesgos de calidad con su tratamiento, además de las principales no conformidades que presenta el proceso de mantenimiento con sus respectivas acciones que se pueden emprender para minimizarlas permitiendo la implantación futura del SGC. Implantar y certificar el sistema traería consigo para hotel numerosos beneficios, dentro de ellos está la elevación de la imagen en el mercado tanto nacional como internacional, incremento de 
las utilidades debido al aumento y repitencia de los clientes, mayor control de los impactos que tiene el proceso en el medio ambiente, prestación de un servicio eficiente lo que elevaría la satisfacción de los cliente, los cuales constituyen la razón de ser de la organización.

En el año 2015 los gastos fueron de 151700 cuc por concepto de compra de equipos como son: neveras, televisores, microwaves, ventiladores, hornos, equipos de clima entre otros y en el 2016 los valores alcanzaron la cifra de 153800 cuc por los mismos conceptos, superando en más de 2000 cuc las del año anterior. Con la correcta realización del plan de mantenimiento y con la responsabilidad e interés que el mismo requiere la institución dejaría de gastar 2000 cuc o más por cada año para reponer los equipos antes mencionados.

\section{Conclusiones}

1. Con la sistematización de los referentes teóricos sobre la gestión de la calidad en los servicios se pudo constatar que la prestación de un servicio con calidad permite satisfacer a los clientes tanto internos como externos así como generar utilidades para la organización.

2. La aplicación parcial del procedimiento seleccionado permitió identificar las insuficiencias en los Servicios Técnicos y proponer acciones de mejora.

\section{Bibliografía}

Abreu, E. L.

2011. Procedimiento para el diseño de un sistema de gestión de la calidad basado en un enfoque de procesos.

Almaguer de Miguel, A.

2015. Propuesta de un plan de mejora de la gestión de la calidad en el Hotel Brisas Covarrubias, Puerto Padre, Las Tunas.

Autores, C. d.

Metodología para un diagnóstico que permita certificar el Sistema de Gestión de la Calidad basado en los requisitos de la NC ISO 9001:2008. Oscar Lucero Moya, Holguín.

Aguilera, M. A. M.

2015. Sistema de Gestión Ambiental en el Hotel Brisas Covarrubias. Universidad de las Tunas

Alfonso, K. J.

2011. Diagnóstico del Sistema de Gestión de la Calidad en el hotel Las Tunas de la cadena Islazul.

Las Tunas

Autores, C. d.

2006. Metodología para un diagnóstico que permita certificar el Sistema de Gestión de la Calidad basado en los requisitos de la NC ISO 001:2008

Cabrera, M. H. R.

2016. Tendencias Internacionales Y Nacionales En Los Sistemas De Gestión. Universidad Y Sociedad. Castellucci, D.

2003. Turismo, Desarrollo y Calidad: hacia un servicio sostenible.

Cols, J. B. S. M. R. Guía para una gestión basada en procesos.

Concepción, I. H.

2007. Metodología para implementar la Gestión de la Calidad. Holguín.

Cubanacán, G. Manual Operativo de la Marca Brisas.

Daniel, A. S.

2005. Procedimiento para realizar estudios de procesos en empresas hoteleras. Camagüey.

Rodríguez, L. T.

2016. Procedimiento Para El Diseño Del Sistema De Gestión De La Calidad En El Proceso Operativo

De Restauración En El Hotel Brisas Covarrubias. Universidad de Las Tunas.

Saturno, P. J.

2011. Tendencias actuales en los sistemas de gestión de la calidad. Tendencias y nuevos conceptos en calidad (2017), from http://www.cmseditorial.com/

Recibido:

$09 / 10 / 2017$

Reenviado:

$08 / 05 / 2018$

Aceptado:

$28 / 02 / 2018$

Sometido a evaluación por pares anónimos 